\title{
Clinical effect of T-SPOT.TB test for the diagnosis of tuberculosis
}

\author{
Yanfen $\mathrm{Ma}^{1 \dagger}$, Ruicheng $\mathrm{Li}^{2+}$, Jinghui Shen ${ }^{3+}$, Longmei $\mathrm{He}^{4}$, Ying $\mathrm{Li}^{5}$, Ning Zhang ${ }^{1}$, Qian $\mathrm{Wu}^{1}$, Jinling Zhang ${ }^{6}$, \\ Jie Zheng ${ }^{7^{*}}$ and Xiaoqin Wang ${ }^{1 *}$
}

\begin{abstract}
Background: The goal of this study was to further investigate the clinical effectiveness of the T-SPOT.TB test in diagnosing tuberculosis (TB), including the effects of T-SPOT.TB test on evaluating diverse TB types and locations.

Methods: We collected 20,332 specimens from patients suspected to have TB. Afterwards, we performed an integrative analysis of T-SPOT.TB results and clinical diagnoses, and evaluated the composition ratio and positive detection rate of the T-SPOT.TB test in various age groups, sample types, and hospital departments. In addition, we compared the spot number and composition rate between latent TB infection (LTBI), active TB infection, and old TB infection groups. The active TB group was then further divided into pulmonary TB (PTB), pulmonary and extrapulmonary TB (PETB), and extrapulmonary TB (EPTB) subgroups, and we evaluated whether there were statistical differences in spot number and composition rate between subgroups.
\end{abstract}

Results: Positive results from the T-SPOT.TB test were found across different age groups, specimen types, and hospital departments. Elderly patient groups, pleural effusion samples, and thoracic surgery departments showed the highest rates of positivity. There were no statistically significant differences in spot number of CFP-10 and ESAT-6 wells between disease groups or active TB subgroups. The composition rate, however, was significantly different when ESAT-6 and CFP-10 wells were double-positive. The spot number and composition rate were statistically different between the three disease groups, but showed no significant differences between the three subgroups of active TB.

Conclusions: The results of T-SPOT. TB test showed differences in LTBI, active TB and old TB. Additionally, a higher spot number level was observed in the active TB group.

Keywords: T cell spot test of tuberculosis infection, Mycobacterium tuberculosis, Active tuberculosis, Clinical diagnosis

\section{Background}

Tuberculosis (TB) is a common chronic disease caused by infection with the facultative intracellular pathogenic bacteria Mycobacterium tuberculosis, and is a serious danger to public health [1]. It has been reported that Mycobacterium tuberculosis (M.TB) can spread hematogenously to various tissues and organs including the lung apices, lymph nodes, spleen, and liver, which has been considered to be one crucial infectious source in

\footnotetext{
* Correspondence: jiezheng@xjtu.edu.cn; pusu294jiaqiao@163.com

†Yanfen Ma, Ruicheng Li and Jinghui Shen contributed equally to this work.

${ }^{7}$ Clinical Research Center, The The First Affiliated Hospital of Xi'an Jiaotong

University, Xi'an 710061, Shaanxi Province, China

'Department of Clinical Laboratory, The First Affiliated Hospital of Xi'an

Jiaotong University, Xi'an 710061, Shaanxi Province, China

Full list of author information is available at the end of the article
}

TB occurrence [2]. Remarkably, there are approximately 10.4 million new cases of $\mathrm{TB}$ and 1.8 million deaths every year around the world [3] and nearly one-third of the population in the world is regarded to be latently infected, according to the World Health Organization's investigation and assessment [4]. Recently, China has been reported to be 1 of 30 high-burden TB countries, due to high TB morbidity [5]. In addition, TB characteristics tend to be concealed by complications and other symptoms because of the growing numbers of elderly patients, increasing patients with drug resistance and extensive immune impairment, causing the slow progression of $\mathrm{TB}$, atypical symptoms and misdiagnosis or missed-diagnosis in clinical examinations [6]. Therefore, it is essential to control TB progression, and undertake early prevention, diagnosis, and treatment.

(C) The Author(s). 2019 Open Access This article is distributed under the terms of the Creative Commons Attribution 4.0 International License (http://creativecommons.org/licenses/by/4.0/), which permits unrestricted use, distribution, and 
Acid-fast staining, TB antibody examination and realtime quantitative PCR have been the predominant methods for $M$. tuberculosis detection in clinical analyses [7]. Unfortunately, these approaches have numerous drawbacks, such as unsatisfactory sensitivity and specificity, high specimen requirements, and susceptibility to immune state [8]. Encouragingly, the T-SPOT.TB assay (an interferon (IFN) $-\gamma$ release assay) is based on detecting secreted IFN- $\gamma$ in $M$. tuberculosis-specific T-cells stimulated by Mycobacterium-specific antigens. These antigens include early secreted antigenic target 6 (ESAT6) and culture filtrate protein 10 (CFP-10), which have been successfully utilized in T-cell effect tests to determine whether $M$. tuberculosis infection exists $[9,10]$. Moreover, accumulating evidence has suggested that this powerful approach can effectively diagnose $M$. tuberculosis infection for patients with or without TB symptoms, and has relatively high sensitivity and specificity [11]. However, a T-SPOT.TB analysis based on a larger sample size has rarely been performed in TB diagnoses, and an evaluation of the T-SPOT.TB test's effects on diverse TB types and locations has not been undertaken.

Here, 20,332 subjects suspected of having TB were enrolled at the First Affiliated Hospital of Xi'an Jiaotong University, and subjected to T-SPOT.TB assay. Subsequently, corresponding comparison analyses including the composition ratio and positive rate in various age groups, sample types, and hospital departments were performed. Moreover, the spot number and composition rate in different disease types (latent $\mathrm{TB}$, active $\mathrm{TB}$, and old TB), and three active TB subgroups (PTB, PETB and EPTB subgroups) were evaluated. This work will contribute to carrying out efficient TB clinical diagnosis and prevention based on T-SPOT.TB test.

\section{Methods \\ Subjects}

In total, 20,332 samples were collected from patients suspected of having TB. The participants' average age was $53.15 \pm 18.16$ years, and they were admitted to the First Affiliated Hospital of Xi'an Jiaotong University from July 2013 to May 2017. Specifically, there were 11, 453 males between 6 months and 96 years of age (Mean age: $54.70 \pm 18.45$ ), and 8879 females between 3 months and 96 years of age (Mean age: $52.53 \pm 17.71$ years). All patients were enrolled according to the following criteria: 1) the subjects were without heart, liver, or kidney diseases, and did not have an HIV infection; 2) patients were suspected to have TB; 3) Patients were not taking any therapies that involved immunosuppression or enhancer medication. The exclusion criteria included: 1) cases lost to follow-up; 2) cases lost to death; 3) cases that were not diagnosed as the TB at end points.
Informed consent was obtained from all participants (informed consent of patients under 16 years of age was obtained from their guardians), and this study was approved by the ethics committee of the First Affiliated Hospital of Xi'an Jiaotong University (NO. XJTU1AF2018LSK-161).

\section{Diagnostic criteria for different groups}

In light of the diagnostic criteria for pulmonary $\mathrm{TB}$ outlined by the Ministry of Health of the People's Republic of China, the included patients were grouped as follows: 1) LTBI: tuberculin test was positive and patients who had no history of the Bacillus Calmette - Guerin (BCG) vaccination; or the test result of TSPOT.TB was positive and there was no clinical manifestation of TB and corresponding evidence from etiology, pathology and imageology. 2) active TB: this required that tubercle bacilli were detected from bacterial cultures or sputum smear, that caseate or Langhans' giant cells were observed by pathological examination, and that antituberculous therapy was effective with relevant imaging support. Moreover, active TB was further divided into three subgroups based on pathological site: pulmonary TB (PTB), pulmonary and extrapulmonary TB (PETB), and extrapulmonary TB (EPTB). 3) old TB: this required that subjects with a history of TB were healed, but pathological changes were found according to the imageological diagnosis, that symptoms of $\mathrm{TB}$ poisoning were not observed and tests from etiology and pathology were negative, and that the patients with these characteristics were diagnosed with "other diseases".

\section{Sample collection and handling}

Peripheral venous blood samples of $5 \mathrm{ml}$ were obtained from patients suspected of having $\mathrm{TB}$ using heparin lithium-anticoagulant tubes. Mononuclear cells were then isolated to prepare a cell suspension. Finally, the TSPOT.TB assay (Oxford Immunotec, Ltd., Abingdon, UK) was performed as follows: briefly, the cell suspension was seeded onto T-SPOT.TB plates and incubated with ESAT-6 (specific antigen), CFP-10 (specific antigen), positive control, or negative control, respectively. A $100 \mu \mathrm{l}$ cell suspension was then added into corresponding microwells, and these were cultured in an incubator with $5 \% \mathrm{CO}_{2}$ at $37^{\circ} \mathrm{C}$. Microwells were then washed four times with phosphate buffer solution (PBS), before $50 \mu \mathrm{l}$ of secondary antibody solution was added into each well, and the assay was incubated for $1 \mathrm{~h}$ at $2-8{ }^{\circ} \mathrm{C}$. Subsequently, $50 \mu \mathrm{l}$ of chromogenic agent was added, and the plate was processed under light avoidance for 7 to 12 min before termination with distilled water. The number of spots were measured, where one spot represented one $\mathrm{T}$ cell which could secrete specific cytokines [12]. The 
final results interpretation was in accordance with following criteria: 1) Results were considered positive in two scenarios: first, if the number of spots in the negative control group was less than 6 , and the spot number in CFP-10 or ESAT- 6 wells was 6 spots greater than that of negative control. Second, if the spot count in the negative control group was 6 to 10, and the CFP-10 or ESAT-6 spot number was more than two times that of the negative control. 2) Results were considered negative if the spot number did not meet the above criteria, and the positive control performed normally. Finally, we performed statistical analyses of spot number and, the spots accounted for the proportion of total spots (composition rate) in different disease types (LTBI, active TB and the old TB) and different active TB subgroups (PTB, PETB and $\mathrm{EPTB})$.

\section{Statistical analysis}

All data analyses were carried out using SPSS software (version 18.0, IBM). A $\chi^{2}$ test was applied to assess comparison analyses. And "inspection rate" (means the percentage of certain samples among all tested samples) was calculated. T-SPOT.TB results showed a skewed distribution and were expressed as the median and interquartile range. Nonparametric tests were used for the comparative analyses of different groups, and $p<0.01$ was considered statistically significant.

\section{Results}

\section{Distribution of T-SPOT.TB results in different ages}

A total of 20,332 patients were distributed into various age groups, of which the childhood group had the fewest individuals (0-6 years old; 68 cases), while the overwhelming majority of patients belonged to the middleaged group (41-65 years old; 9735 cases). Interestingly, the positive rate of tests was highest in the elderly group (41.91\%, Table 1). In addition, positive rate of whole blood was $36.60 \%$ (Table 1).

\section{Distribution of T-SPOT.TB results in different hospital departments}

The samples selected in this work covered 29 hospital departments, such as respiration, rheumatology, or urology departments (Table 2). We noted that the highest inspection rate was in the respiration department (8261 cases; $40.63 \%)$, followed by the rheumatology department (2924 cases; $14.38 \%$ ). The pain department exhibited the lowest test rate (10 cases; $0.05 \%)$. Additionally, in terms of positive rates of tests, the thoracic surgery department was in the lead (53.20\%), followed by the general surgical department $(50.00 \%)$. The lowest positive rate of tests was $5.26 \%$ for the pediatric department.

\section{Comparisons of spots numbers and composition rate in three groups}

The findings of T-SPOT.TB tests suggested that there were 7464 positive cases, out of a total of 20,332 cases. After removing undiagnosed (691 cases) and non-whole blood specimens (103 cases), the remaining 6760 cases were divided into three groups, including LTBI (4612 cases), active TB (1814 cases), and old TB groups (334 cases). The spot number types of T-SPOT.TB tests were as follows: ESAT- 6 unique positive, CFP-10 unique positive; ESAT-6 and CFP-10 double positive; total spot number of ESAT-6 and CFP-10. A comparative analysis implied that the spot number of ESAT- 6 and CFP-10 wells were not significantly different between each disease group $(p>0.05)$, but the composition rate in each group was dramatically different when ESAT-6 and CFP-10 microwells were double positive $(p<0.01)$ (Table 3). For intergroup comparative analyses, we found that the spot number and composition rate were statistically different among three groups $(p<0.01)$. CFP-10 and ESAT-6 double positive rates in active TB (79.16\%) were higher than LTBI $(56.15 \%)$ and old TB (66.77\%) (Table 3).

Table 1 The results of T-SPOT.TB in 20,332 patients from different age groups and specimen types

\begin{tabular}{|c|c|c|c|c|c|c|}
\hline Items & Cases & Proportion (\%) & Positive cases & Proportion (\%) & Positive proportion (\%) & Positive rate (\%) \\
\hline \multicolumn{7}{|l|}{ Age groups } \\
\hline 0-6years old & 68 & 0.33 & 3 & 0.01 & 0.04 & 4.41 \\
\hline 7-17 years old & 410 & 2.02 & 65 & 0.32 & 0.87 & 15.85 \\
\hline $18-40$ years old & 4247 & 20.89 & 1441 & 7.09 & 19.31 & 33.93 \\
\hline 41-65 years old & 9735 & 47.88 & 3494 & 17.18 & 46.81 & 35.89 \\
\hline$\geq 66$ years old & 5872 & 28.88 & 2461 & 12.10 & 32.97 & 41.91 \\
\hline Total & 20,332 & 100.00 & 7464 & 36.71 & 100.00 & 36.71 \\
\hline \multicolumn{7}{|l|}{ Specimen types } \\
\hline Whole blood & 20,107 & 98.89 & 7361 & 36.20 & 98.61 & 36.60 \\
\hline Total & 20,332 & 100.00 & 7464 & 36.71 & 100.00 & 36.71 \\
\hline
\end{tabular}


Table 2 The results of T-SPOT.TB in 20,332 patients from different clinical departments

\begin{tabular}{|c|c|c|c|c|c|c|}
\hline Departments & Cases & Proportion (\%) & Positive cases & Propotion (\%) & Positive proportion (\%) & Positive rates (\%) \\
\hline Pain & 10 & 0.05 & 4 & 0.02 & 0.05 & 40.00 \\
\hline Cardiovascular surgery & 25 & 0.12 & 9 & 0.04 & 0.12 & 36.00 \\
\hline Ear-nose-throat & 39 & 0.19 & 19 & 0.09 & 0.25 & 48.72 \\
\hline Emergency & 47 & 0.23 & 13 & 0.06 & 0.17 & 27.66 \\
\hline General surgery & 50 & 0.25 & 25 & 0.12 & 0.34 & 50.00 \\
\hline Gynaecology and obstetrics & 51 & 0.25 & 24 & 0.12 & 0.32 & 47.06 \\
\hline Breast Surgery & 53 & 0.26 & 19 & 0.09 & 0.25 & 35.85 \\
\hline Traditional Chinese medicine & 75 & 0.37 & 33 & 0.16 & 0.44 & 44.00 \\
\hline Abdominal tumor surgery & 79 & 0.39 & 32 & 0.16 & 0.43 & 40.51 \\
\hline Peripheral vascular & 79 & 0.39 & 37 & 0.18 & 0.50 & 46.84 \\
\hline Radiotherapy & 83 & 0.41 & 38 & 0.19 & 0.51 & 45.78 \\
\hline Dermatology & 84 & 0.41 & 26 & 0.13 & 0.35 & 30.95 \\
\hline Hepatobiliary surgery & 99 & 0.49 & 39 & 0.19 & 0.52 & 39.39 \\
\hline Pediatrics & 152 & 0.75 & 8 & 0.04 & 0.11 & 5.26 \\
\hline Urinary surgery & 157 & 0.77 & 77 & 0.38 & 1.03 & 49.04 \\
\hline Central ICU & 180 & 0.89 & 46 & 0.23 & 0.62 & 25.56 \\
\hline Medical oncology & 208 & 1.02 & 69 & 0.34 & 0.93 & 33.17 \\
\hline Endocrinology & 358 & 1.76 & 154 & 0.76 & 2.07 & 43.02 \\
\hline Orthopedics & 365 & 1.80 & 172 & 0.85 & 2.31 & 47.12 \\
\hline Cadre's ward & 381 & 1.87 & 167 & 0.82 & 2.24 & 43.83 \\
\hline Cardiology & 478 & 2.35 & 214 & 1.05 & 2.87 & 44.77 \\
\hline Neurology & 518 & 2.55 & 187 & 0.92 & 2.51 & 36.10 \\
\hline Thoracic surgery & 594 & 2.92 & 316 & 1.55 & 4.24 & 53.20 \\
\hline Hematology & 734 & 3.61 & 170 & 0.84 & 2.28 & 23.16 \\
\hline Digestive System & 778 & 3.83 & 304 & 1.50 & 4.08 & 39.07 \\
\hline Infectious & 1203 & 5.92 & 377 & 1.85 & 5.06 & 31.34 \\
\hline Nephrology & 2267 & 11.15 & 853 & 4.20 & 11.44 & 37.63 \\
\hline Rheumatology & 2924 & 14.38 & 705 & 3.47 & 9.46 & 24.11 \\
\hline Respiration & 8261 & 40.63 & 3327 & 16.36 & 44.57 & 40.27 \\
\hline Total & 20,332 & 100.00 & 7464 & 36.71 & 100.00 & 36.71 \\
\hline
\end{tabular}

Comparisons of spot numbers and composition rate in three subtypes of active TB

The active TB group contained three subgroups (PTB: 547 cases; PETB: 199 cases; EPTB: 1068 cases) as mentioned above. The results revealed that the spot number for CFP-10 and ESAT-6 microwells in each subgroup were not significantly different $(p>0.05)$, but the composition rate in each subtype was significantly different when CFP-10 and ESAT-6 microwells were double positive $(p<$ $0.01)$. Moreover, there were no significant differences in terms of the numbers of spots, and the composition rate among three subgroups $(p>0.05)$ (Table 4$)$.

\section{Discussion}

Overwhelming evidence has suggested that morbidity, misdiagnosis and missed diagnosis of TB has remained at high levels over the past few years. This is primarily due to the prevalence of acquired immune deficiency syndrome (AIDS), growing population mobility, and increasing $M$. tuberculosis drug-resistant mutants $[13,14]$. Although there has been a variety of approaches for diagnosing $M$. tuberculosis, the sensitivity and specificity of these assays is not satisfactory, posing enormous challenges for clinical diagnosis [15]. Fortunately, TSPOT.TB is a new, promising diagnosis method, that has been broadly applied in clinical research. This test detects IFN- $\gamma$ released from T-cells that are exposed to the M. tuberculosis-specific antigens ESAT- 6 and CFP10 , to measure T-cell number and determine $M . T B$ infection status [16]. In addition, this method shows satisfactory sensitivity (78.4\%) and greater specificity (59.0 to 93.0\%) than previous assays [17-20]. Moreover, a 
Table 3 Comparative results of the numbers of spots and composition in three groups

\begin{tabular}{|c|c|c|c|c|c|}
\hline Spot types & LTBI & Active TB infection & Old TB infection & $X^{2} / P$ & $Z / P$ \\
\hline \multicolumn{6}{|l|}{ ESAT-6 unique positive } \\
\hline Cases & 1281 & 270 & 69 & & \\
\hline Composition rate & 27.78 & 14.88 & 20.66 & $120.844 /<0.001$ & \\
\hline Median spot number & 12.00 & 20.00 & 16.00 & & $57.080 /<0.001$ \\
\hline Spot number interquartile range & $8.00,23.00$ & $12.00,36.25$ & $8.50,26.50$ & & \\
\hline \multicolumn{6}{|l|}{ CFP-10 unique positive } \\
\hline Cases & 741 & 108 & 42 & & \\
\hline Composition rate & 16.07 & 5.96 & 12.57 & $116.473 /<0.001$ & \\
\hline Median spot number & 11.00 & 17.00 & 19.50 & & $19.355 /<0.001$ \\
\hline Spot number interquartile range & $7.00,20.00$ & $9.00,31.75$ & $7.00,32.25$ & & \\
\hline \multicolumn{6}{|l|}{ ESAT-6 and CFP-10 double positive } \\
\hline Cases & 2590 & 1436 & 223 & & \\
\hline Composition rate & 56.15 & 79.16 & 66.77 & $297.397 /<0.001$ & \\
\hline ESAT-6/median spot number & 24.00 & 44.00 & 29.00 & & $320.640 /<0.001$ \\
\hline ESAT-6/ Spot number interquartile range & $12.00,46.00$ & $24.00,80.00$ & $14.00,60.00$ & & \\
\hline CFP-10/ median spot number & 24.00 & 46.00 & 26.00 & & $320.728 /<0.001$ \\
\hline CFP-10 (Spot number interquartile range) & $12.00,47.00$ & $23.00,89.00$ & $15.00,54.00$ & & \\
\hline \multicolumn{6}{|l|}{ Total spot number } \\
\hline Cases & 4612 & 1814 & 334 & & \\
\hline Composition rate & 100.00 & 100.00 & 100.00 & & \\
\hline ESAT-6/ median spot number & 15.00 & 36.00 & 20.00 & & $635.730 /<0.001$ \\
\hline ESAT-6/(Spot number Interquartile range) & $7.00,33.00$ & $17.00,70.00$ & $9.00,43.00$ & & \\
\hline CFP-10/median spot number & 12.00 & 35.00 & 19.00 & & $535.277 /<0.001$ \\
\hline CFP-10 (Spot number interquartile range) & $4.00,30.00$ & $11.00,75.00$ & $6.00,40.25$ & & \\
\hline
\end{tabular}

TB: tuberculosis; LTBI: latent TB infection

previous study has highlighted that active TB, LTBI, and old TB can all be detected using this method [21]. Another study has revealed that specificity is correlated with LTBI and old TB [22]. Here, our work aimed to explore the practical application effectiveness of $\mathrm{T}$ SPOT.TB across various TB types and locations and analyze this assay's performance in a large dataset.

Our findings suggest that T-SPOT.TB positive tests are distributed in different age groups, and that positive rates increase with age. A plausible explanation for this finding is that the probability of exposure to M.TB increased with age, which increased the risk for TB. This further validated the fact that there is a large population of patients with LTBI and old TB in China. The number of LTBI (4612 cases) was higher than active TB (1814 cases), which is consistent with other research [23]. Additionally, previous investigations have demonstrated that the T-SPOT.TB technique is also successfully employed in specimens other than blood such as pleural fluid, ascitic fluid, and cerebrospinal fluid [24]. Furthermore, the test results of these specimens provided relatively higher diagnostic value than a whole blood test [23]. However,
Keng et al. stated that T-SPOT.TB values for other specimen types remain to be determined, because of numerous uncertainties and large bias [25].

There were 29 clinical departments in our analysis. Although the highest inspection rate emerged in the respiration department (8261 cases), followed by the rheumatology department (2924 cases), the thoracic surgery department (53.20\%) displayed the highest positive rate. Moreover, the results showed that there were 13 departments where the positive rate was higher than the respiration department. In addition, we found that active TB (1814 cases) included PTB (547 cases), PETB (547 cases), and ЕРТВ (1068 cases) groups, suggesting that EPTB showed a predominant infection rate. It is because EPTB cases were characterized by complicated etiologies, atypical clinical presentations, difficulties in diagnosis, and therapy and irreversible damage, which will lead to the high infection rate.

Notably, we first conducted a spot number analysis of T-SPOT.TB tests in terms of TB infectious types and locations in three TB groups, including active TB, LTBI and old TB. The results suggested that the number of 
Table 4 Comparative results of the numbers of spots and composition in three subgroups

\begin{tabular}{|c|c|c|c|c|c|}
\hline Spot types & PTB & PETB & EPTB & $x^{2} / P$ & $Z / P$ \\
\hline \multicolumn{6}{|l|}{ ESAT-6 unique positive } \\
\hline Cases & 89 & 27 & 154 & & \\
\hline Composition rate & 16.27 & 13.57 & 14.42 & $1.284 / 0.526$ & \\
\hline Median spot number & 22.00 & 21.00 & 17.00 & & $5.655 / 0.059$ \\
\hline Spot number interquartile range & $14.00,42.00$ & $12.00,39.00$ & $10.00,31.50$ & & \\
\hline \multicolumn{6}{|l|}{ CFP-10 unique positive } \\
\hline Cases & 27 & 5 & 76 & & \\
\hline Composition rate & 4.94 & 2.51 & 7.12 & $7.798 / 0.020$ & \\
\hline Median spot number & 18.00 & 9.00 & 16.50 & & $0.014 / 0.993$ \\
\hline Spot number interquartile range & $9.00,30.00$ & $8.50,125.00$ & $9.25,33.75$ & & \\
\hline \multicolumn{6}{|l|}{ ESAT-6 and CFP-10 double positive } \\
\hline Cases & 431 & 167 & 838 & & \\
\hline Composition rate & 78.79 & 83.92 & 78.46 & $3.091 / 0.213$ & \\
\hline ESAT-6/ Median spot number & 44.00 & 42.00 & 44.00 & & $1.516 / 0.469$ \\
\hline ESAT-6/(Spot number interquartile range) & $25.00,83.00$ & $24.00,87.00$ & $23.00,78.00$ & & \\
\hline CFP-10/ Median spot number & 42.00 & 53.00 & 47.00 & & $3.337 / 0.189$ \\
\hline CFP-10 (Spot number interquartile range) & $22.00,86.00$ & $29.00,94.00$ & $23.00,89.00$ & & \\
\hline \multicolumn{6}{|l|}{ Total spot number } \\
\hline Cases & 547 & 199 & 1068 & & \\
\hline Composition rate & 100.00 & 100.00 & 100.00 & & \\
\hline ESAT-6/ Median spot number & 39.00 & 38.00 & 34.00 & & $7.061 / 0.029$ \\
\hline ESAT-6/(Spot number interquartile range) & $19.00,73.00$ & $20.00,82.00$ & $15.00,67.00$ & & \\
\hline CFP-10/ Median spot number & 30.00 & 42.00 & 36.00 & & $5.315 / 0.070$ \\
\hline CFP-10 (Spot number interquartile range) & $11.00,67.00$ & $14.00,84.00$ & $11.00,76.75$ & & \\
\hline
\end{tabular}

TB: tuberculosis; PTB: pulmonary TB; PETB: pulmonary and extrapulmonary TB; EPTB extrapulmonary TB

spots in ESAT-6 and CFP-10 wells were not significantly different between each disease group, but the composition rate in each group was dramatically different in ESAT-6 and CFP-10 double positive microwells $(p<$ 0.01 ). Additionally, we found that the spot number and composition rate were statistically different among the three groups. In this study, we highlighted that ESAT-6 and CFP-10 tests were independent, while spot numbers in each disease group were not statistically different. Therefore, we inferred that there was no significant difference in subjects' responses to the two specific antigens. In addition, comparative analyses among the three groups implied that the spot number in active TB was higher than LTBI and old TB. Many researchers have claimed that although the positive correlation between spot numbers and TB activity has not been determined, increased spot counts are observed when TB activity was stronger [26, 27], an observation that is consistent with our results. We thus infer that higher spot counts can distinguish active TB from LTBI and old TB. In addition, previous work has evaluated the application values of $\mathrm{T}$ SPOT.TB in detecting PTB and EPTB, and found that negative T-SPOT.TB results could be considered vital predictors for TB diagnosis [28]. However, the relationship between spot number and these two types of $\mathrm{TB}$ (PTB and EPTB) has not been investigated [29, 30]. Here, we found that there were no significant differences in the number of spots and composition rates for intraor inter-subgroups analyses $(p>0.01)$, which suggests that the identification of PTB, PETB, and ЕPTB on the basis of the spot number level is not satisfactory.

There were several limitations in this work. Previous studies have pointed out that the immune status of subjects may affect the results of T-SPOT.TB. In this study, we did not consider numerous pivotal factors associated with the patients' immune states, including T-cell activity, nutritional status, additional complications, body mass index, and smoking and drinking. This likely created a mixed basis for results and may influence further stratified analyses in results of T-SPOT.TB. Moreover, a comprehensive analysis that combines T-SPOT.TB test results, TB etiology, imaging, and histopathology with area-specific TB prevalence is still needed for TB detection and prevention. 


\section{Conclusions}

In conclusion, T-SPOT.TB test proved to be valuable for distinguishing between LTBI, active TB and old TB. In addition, a higher spot number level was observed in the active TB patient group, but T-SPOT.TB test showed low specificity for the diagnosis of different active TB subtypes.

\section{Abbreviations}

AIDS: Acquired immune deficiency syndrome; CFP-10: Culture filtrate protein 10; EPTB: Extrapulmonary TB; ESAT-6: Early secreted antigenic target 6; LTBI: Latent TB infection; PETB: Pulmonary and extrapulmonary TB; PTB: Pulmonary TB; TB: Tuberculosis

\section{Acknowledgements}

None.

\section{Authors' contributions}

Conception and design of the research: $Y M, X W$; acquisition of data: RL, JS, JZ1; analysis and interpretation of data: YM, LH, YL; statistical analysis: NZ, QW, JZ2; obtaining funding: XW; drafting the manuscript: $Y M, R L$, JS; revision of manuscript for important intellectual content: XW, JZ1. All authors read and approved the final manuscript.

\section{Funding}

This work was supported by the International Science and Technology Cooperation and Exchange Program of Shaanxi Province (Grant No. 2015KW045) and the Clinical Research Awards of the First Affiliated Hospital of Xi'an Jiaotong University, China. (No. XJTU1AF-CRF-2016-019 and XJTU1AF-CRF2018-013). These funding bodies had no role in the design of the study and collection, analysis, and interpretation of data and in writing the manuscript.

\section{Availability of data and materials}

The datasets used during the current study available from the corresponding author on reasonable request.

\section{Ethics approval and consent to participate}

This study was approved by the Ethics Committee of The First Affiliated Hospital of Xi'an Jiaotong University. Based on the approval of Ethics Committee of the First Affiliated Hospital of Xi'an Jiaotong University, the informed consents obtained from the patients was verbal due to there was no health damage or information exposure to the participants in this retrospective study; and for patients $\leq 16$, we directly communicated with the guardian over the phone and obtained their consent.

\section{Consent for publication}

Not applicable.

\section{Competing interests}

The authors declare that they have no competing interests.

\footnotetext{
Author details

'Department of Clinical Laboratory, The First Affiliated Hospital of Xi'an Jiaotong University, Xi'an 710061, Shaanxi Province, China. ${ }^{2}$ Department of Clinical Laboratory, The Second Affiliated Hospital of Air Force Medical University, Xi'an 710038, Shaanxi Province, China. ${ }^{3}$ Department of Clinical Laboratory, Xi'an Central Hospital, Xi'an 710003, Shaanxi Province, China. ${ }^{4}$ Department of Clinical Laboratory, Shaanxi Provincial Hospital of Traditional Chinese Medicine, Xi'an 710061, Shaanxi Province, China. ${ }^{5}$ Department of Clinical Laboratory, Shaanxi KangFu Hospital, Xi'an 710061, Shaanxi Province, China. 'Department of Clinical Laboratory, Xi'an Encephalopathy Hospital, Xi'an 710061, Shaanxi Province, China. ${ }^{7}$ Clinical Research Center, The The First Affiliated Hospital of Xi'an Jiaotong University, Xi'an 710061, Shaanxi Province, China.
}

Received: 19 December 2018 Accepted: 25 October 2019

Published online: 21 November 2019

\section{References}

1. Shastri M, Shukla S, Chong WC, Dua K, Peterson GM, Patel R, Hansbro P, Eri $\mathrm{R}$, O'Toole R. Role of oxidative stress in the pathology and Management of Human Tuberculosis. Oxidative Med Cell Longev. 2018.

2. Kim PM, Lee JJ, Choi D, Eoh H, Hong YK: Endothelial lineage-specific interaction of Mycobacterium tuberculosis with the blood and lymphatic systems. Tuberculosis 2018, 111:1

3. Holmes KK, Bertozzi S, Bloom BR, Jha P. Major Infectious Diseases. 3rd edition. Washington (DC): The International Bank for Reconstruction and Development / The World Bank; 2017 Nov. Chapter 1.

4. Zumla A, George A, Sharma V, Herbert RH, Oxley A, Oliver M. The WHO 2014 global tuberculosis report--further to go. Lancet Glob Health. 2015:3(1):e10-2.

5. Lu D, Chen C, Yu S, Chen S. Diagnosis of Tuberculous meningitis using a combination of peripheral blood T-SPOT.TB and cerebrospinal fluid interferon-gamma detection methods. Lab Med. 2016;47(1):6-12.

6. Shah M, Reed C. Complications of tuberculosis. Curr Opin Infect Dis. 2014; 27(5):403-10.

7. Lee E, Holzman RS. Evolution and current use of the tuberculin test. Clin Infect Dis. 2002:34(3):365-70.

8. Auguste P, Tsertsvadze A, Pink J, Court R, McCarthy N, Sutcliffe P, Clarke A. Comparing interferon-gamma release assays with tuberculin skin test for identifying latent tuberculosis infection that progresses to active tuberculosis: systematic review and meta-analysis. BMC Infect Dis. 2017; 17(1):200

9. Zellweger JP, Zellweger A Fau - Ansermet S, Ansermet S Fau - de Senarclens B, de Senarclens B Fau - Wrighton-smith P, Wrighton-smith P: contact tracing using a new T-cell-based test: better correlation with tuberculosis exposure than the tuberculin skin test. Int J Tuberc Lung Dis 2005, 9(11):1242-1247

10. Stout JE, Wu Y, Ho CS, Pettit AC, Feng PJ, Katz DJ, Ghosh S, Venkatappa T, Luo R. Evaluating latent tuberculosis infection diagnostics using latent class analysis. Thorax. 2018;73(11):1062-70.

11. Bastian I, Coulter C. Position statement on interferon-gamma release assays for the detection of latent tuberculosis infection. Commun Dis Intell Q Rep. 2017:41(4):E322-36.

12. Kobashi Y, Mouri K, Yagi S, Obase Y, Miyashita N, Oka M. Transitional changes in T-cell responses to Mycobacterium tuberculosis-specific antigens during treatment. J Infect. 2009;58(3):197-204.

13. Liu Q, Yu Y-X, Wang X-J, Wang Z, Wang Z. Diagnostic accuracy of interleukin-27 between tuberculous pleural effusion and malignant pleural effusion: a meta-analysis. Respiration. 2018:95:469-77.

14. Rahimkhani M, Einollahi N, Daneshvar HK, Dashti N. Survey of serum procalcitonin in cirrhotic patients. Acta Medica Iranica. 2013:153-6.

15. Zhang L, Shi X, Zhang Y, Zhang Y, Huo F, Zhou B, Deng G, Liu X. Analysis of Factors Influencing Diagnostic Accuracy of T-SPOT.TB for Active Tuberculosis in Clinical Practice. Sci Rep. 2017;7(1):7764.

16. Zellweger J-P, Sotgiu G, Block M, Dore S, Altet N, Blunschi R, Bogyi M, Bothamley G, Bothe C, Codecasa L. Risk assessment of tuberculosis in contacts by IFN- $\gamma$ release assays. A tuberculosis network European trials group study. Am J Respir Crit Care Med. 2015;191(10):1176-84.

17. Du F, Xie L, Zhang Y, Gao F, Zhang H, Chen W, Sun B, Sha W, Fang Y, Jia H, et al. Prospective Comparison of QFT-GIT and T-SPOT.TB Assays for Diagnosis of Active Tuberculosis. Sci Rep. 2018;8(1):5882.

18. Mandalakas AM, Highsmith HY, Harris NM, Pawlicka A, Kirchner HL. T-SPOT. TB Performance in Routine Pediatric Practice in a Low TB Burden Setting. Pediatr Infect Dis J. 2018:37(4):292-7.

19. Sargin G, Senturk T, Ceylan E, Telli M, Cildag S, Dogan H. TST, QuantiFERONTB gold test and T-SPOT.TB test for detecting latent tuberculosis infection in patients with rheumatic disease prior to anti-TNF therapy. Tuberk Toraks. 2018;66(2):136-43.

20. Takasaki J, Manabe T, Morino E, Muto Y, Hashimoto M, likura M, Izumi S, Sugiyama H, Kudo K. Sensitivity and specificity of QuantiFERON-TB gold plus compared with QuantiFERON-TB gold in-tube and T-SPOT.TB on active tuberculosis in Japan. J Infect Chemother. 2018;24(3):188-92.

21. Pooran A, Booth H, Miller RF, Scott G, Badri M, Huggett JF, Rook G, Zumla A Dheda K. Different screening strategies (single or dual) for the diagnosis of suspected latent tuberculosis: a cost effectiveness analysis. BMC pulmonary medicine. 2010;10(1):7 
22. Gao L, Lu W, Bai L, Wang X, Xu J, Catanzaro A, Cardenas V, Li X, Yang Y, Du $J$, et al. Latent tuberculosis infection in rural China: baseline results of a population-based, multicentre, prospective cohort study. Lancet Infect Dis. 2015;15(3):310-9.

23. Pan L, Liu F, Zhang J, Yang X, Zheng S, Li J, Jia H, Chen X, Gao M, Zhang Z: Interferon-Gamma Release Assay Performance of Cerebrospinal Fluid and Peripheral Blood in Tuberculous Meningitis in China. BioMed Research International,2017,(2017-02-20) 2017, 2017(3):8198505.

24. Pan LLF, Zhang J, et al. Interferon-gamma release assay performance of cerebrospinal fluid and peripheral blood in Tuberculous meningitis in China. Biomed Res Int. 2017;2017:8198505.

25. Keng L-T, Shu C-C, Chen JY-P, Liang S-K, Lin C-K, Chang L-Y, Chang C-H, Wang J-Y, Yu C-J, Lee L-N. Evaluating pleural ADA, ADA2, IFN- $\gamma$ and IGRA for diagnosing tuberculous pleurisy. J Infect. 2013;67(4):294-302.

26. Tavares SM, Junior Wde L, Lopes ESMR. Normal lymphocyte immunophenotype in an elderly population. Rev Bras Hematol Hemoter. 2014;36(3):180-3.

27. Xu JC, Li ZY, Chen XN, Shi CL, Wu MY, Chen H, Zhu XY, Song HF, Wu MJ, Xu P. More significance of TB-IGRA except for the diagnose of tuberculosis. LID. J Clin Lab Anal. 2018;32(1). https://doi.org/10.1002/jcla.22183, https://doi.org/ 10.1002/jcla.22183.

28. Nguyen DT, Teeter LD, Graves J, Graviss EA. Characteristics associated with negative interferon- $\gamma$ release assay results in culture-confirmed tuberculosis patients, Texas, USA, 2013-2015. Emerg Infect Dis. 2018;24(3):534.

29. Lee YM, Kim SM, Park SJ, Lee SO, Choi SH, Kim YS, Woo JH, Kim SH. Factors associated with a strong response to the T-SPOT.TB in patients with Extrapulmonary tuberculosis. Infect Chemother. 2014:46(4):248-52.

30. Pan L, Jia H, Liu F, Sun H, Gao M, Du F, Xing A, Du B, Sun Q, Wei R, et al. Risk factors for false-negative T-SPOT.TB assay results in patients with pulmonary and extra-pulmonary TB. J Inf Secur. 2015;70(4):367-80.

\section{Publisher's Note}

Springer Nature remains neutral with regard to jurisdictional claims in published maps and institutional affiliations.

Ready to submit your research? Choose BMC and benefit from:

- fast, convenient online submission

- thorough peer review by experienced researchers in your field

- rapid publication on acceptance

- support for research data, including large and complex data types

- gold Open Access which fosters wider collaboration and increased citations

- maximum visibility for your research: over 100M website views per year

At $\mathrm{BMC}$, research is always in progress.

Learn more biomedcentral.com/submissions 